\title{
OBJECT-BASED CHANGE DETECTION ON ACACIA XANTHOPHLOEA SPECIES DEGRADATION ALONG LAKE NAKURU RIPARIAN RESERVE
}

\author{
A. Osio ${ }^{a, *}$, S. Lefèvre ${ }^{b}$ \\ a Technical University of Kenya, Nairobi, Kenya \\ ${ }^{\mathrm{b}}$ Univ. Bretagne Sud, UMR 6074, IRISA, F-56000 Vannes, France
}

KEY WORDS: OBIA, SAR, Change Detection, Flood mapping

\begin{abstract}
:
Automated mapping of heterogeneous riparian landscape is of high interest to assess our planet. Still, it remains a challenging task due to the occurrence of flooded vegetation. While both optical and radar images can be exploited, the latter has the advantage of being independent acquisition conditions. However, and despite their popularity, the threshold-based approaches commonly used present some drawbacks such as not taking into account the spatial context and providing mixed pixels within class boundaries. In this study, we propose a novel methodology to avoid such issues by using an object-based image analysis approach on polarimetric radar data. We use our workflow to map the degrading Acacia X. Species along lake Nakuru Riparian reserve, and obtain highly-accurate results.
\end{abstract}

\section{INTRODUCTION}

Wetlands are important in regulating both the aquatic and benthic ecosystems. In recent time, Lake Nakuru situated along Kenya's great Rift valley has been facing challenges due to the rising water levels (Onywere et al., 2013), hence causing destruction of the National Park's natural and cultural features. Amongst the natural features that have been gradually affected by the floods, and that need to be monitored, is the Acacia xanthophloea tree species which is an important feed for the giraffes and vervet monkeys in the park (Pellew, 1983). However, detecting and mapping flooded areas remains particularly challenging when the target vegetation or trees are immersed in water.

Previous studies have engaged optical and SAR imageries and their derivatives to extract spatial information from riparian reserves. Wetland classification based on optical imagery remains limited due to heavy cloud cover (Amani and Mobasheri, 2015). Conversely, SAR sensors can provide observations irrespective of time of the day or night, penetrating through cloud cover, hence producing images of high quality and integrity (Anusha and Bharath 2020). However, their use in submerged vegetation classification mostly relies on pixel-based thresholding(Schumann et al., 2010) Such a strategy does not take care of the contextual information of the pixels at the land cover class boundaries, thus leading to low classification accuracies.

Thus, the goal of our study is to use time series of Sentinel-1 (VVVH) Synthetic Aperture Radar (SAR) with Single Look Complex (SLC) acquisition modes from 2014 to 2020 within an ObjectBased Image Analysis (OBIA) framework (Franklin and Ahmed, 2017) in order to detect changes in Acacia Xanthophloea degradation along Lake Nakuru Riparian Reserve. Although previous studies have already used S1 Ground Range detected products in flood mapping (Osio et al., 2020), few studies have involved S1 SLC polarimetric SAR products together with an object-level analysis for flood mapping.

\footnotetext{
${ }^{*}$ Corresponding author.
}

\section{MATERIAL \& METHODS}

\subsection{Study Area}

Lake Nakuru National park is located in Nakuru County, 170 kms from Nairobi. Its bottom initially before the recent flooding was about 1756 metres above sea level while the surface of the water at $1758.5 \mathrm{~m}$ above sea level. The altitude ranges from 1760-2080m above sea level. Mean annual rainfall ranges between $876 \mathrm{~mm}$ and $1050 \mathrm{~mm}$ and has an inherent bimodal pattern. The long rains start in March and end in June while the short rains occur between October and December. Mean daily minimum and maximum temperatures fluctuate between $8.2^{\circ} \mathrm{C}$ and $25.6^{\circ} \mathrm{C}$. Lake Nakuru has no outlets and hence evaporation is the only factor that accounts for water loss. Four seasonal rivers feed the lake, ie Lamurdiak, Enjoro, Enderit and Enjoro. The soils are volcanic and shallow in nature. Underneath the open grasslands were soils and ashes which were well drained, friable to sandy clay loams that supported the grasslands in the park. Siltation from sand harvesting contributes to the fluctuation of the water depth in the lake. However, recent flooding of the lake are hypothesized to have been caused by additional factors i.e climate i, change drivers and movement of tectonic plates.

\subsection{Data}

We consider Dual polarized Single Look Complex SAR, all from ascending orbits with phase information between two cross polarized channels, i.e. VV and VH respectively. SAR data were acquired at both wet and dry seasons, from Fall 2015 to Summer 2020 (12 dates). All the data were in the same datum and projection i.e. WGS 84 / UTM Zone 36N.

\subsection{Workflow}

The workflow designed in this study aims to apply an OBIA methodology for change detection on SAR data. It has the following features: i) generating a baseline image using Landsat5TM captured in 2010, ii) extracting features from SAR data, iii) constructing an OBIA ruleset for SAR data, iv) performing a multitemporal map analysis. We now explain the full process by providing details for each successive step. 


\section{Sentinel 1 SLC Preprocessing}

Sentinel 1 Single Look Complex (SLC) of time series preprocessing is conducted as follows: i) S-1 top split into bursts; ii) orbit file alignment; iii) radiometric calibration; iv) multi-date $\mathrm{S}-1$ back-geocoding for image stacking; v) S1 top debursting; vi) AOI subsetting; vi) $\mathrm{C} 2$ covariance matrix extraction; vii) multilooking: viii) refined Lee speckle filtering; ix) range Doppler terrain correction; x) S-1 SLC stack imagery generation.

\section{ALOS PALSAR Preprocessing}

We consider a single date of ALOS PALSAR of L-Band and $\mathrm{HH}+\mathrm{HV}$ channels with a $6.5 \mathrm{~m}$ ground resolution captured in ascending mode from an incident angle of 38.7 degrees. The image was captured in September 2007 and preprocessed using the following steps: i) radiometric calibration; ii) multi-looking using 1 Range look and 7 Azimuth looks; iii) speckle filtering using refined Lee filter; iv) ALOS deskewing for geometric corrections; v) terrain correction; vi) application of land/sea mask for extraction of AOI.

\section{Landsat 5TM Preprocessing}

Landsat 5TM collected in 2010 was used as a baseline map since it was still depicting the state of all riparian classes along the Lake. The steps engaged here were: i) extraction of AOI of the study area; ii) atmospheric corrections done by conversion of DN values to TOA reflectance; iii) pansharpening to $15 \mathrm{~m}$ spatial resolution using Ehlers fusion technique; iv) production of a false colour image RGB using Band 4 (0.73-0.9um), Band 3 (0.63-0.69um) and Band 2 (0.52-0.60um). The latter combination make deciduous trees such as acacia forest appearing bright red in colour, urban area cyan while soil dark to light brown (see Fig. 5).

\section{Sentinel 1 SLC Data Analysis}

From the preprocessed S-1 SLC single date images, we generate bands $\mathrm{C} 11, \mathrm{C} 22, \mathrm{C} 12 \mathrm{REAL}$ and C12IMAG using $2 * 2$ (C2) matrix image in ESA SNAP toolbox (Lee and Pottier, 2017). The spectral bands derived from $C 2$ matrix based single date images consist in radar-based vegetation indices, namely Radar Vegetation Index (RVI), Dual Pol Vegetation Index (DpRVI), Polarimetric Radar Vegetation Index (PRVI) and Degree of Polarization (DOP) for Dual Polarimetric SAR. Time series temporal profiles were then generated from the multi-date stack S1 SLC series built from datasets ranging from 2014 to 2015 as shown in Fig. 1 and Fig. 2. Bands with the highest backscatter response with other additional variables were then used for further data analysis.

\section{ALOS PALSAR Data analysis}

The following bands were derived from ALOS PALSAR image captured in 2007 before the floods with $6.5 \mathrm{~m}$ ground Resolution. Bands $\mathrm{HH}$ and $\mathrm{HV}$ were converted into $\sigma(\mathrm{HH})$ and $\sigma(\mathrm{HV})$. These bands were transited to eCognition Developer 10.1 where additional variables such as the PCA1, PCA2 mean and standard deviation were added into the classification. Other variables include geometric features such as Border Index and Ecliptic fit. Classifiers appended on the classification include K-NN, Naive Bayes, Decision Tree (DT) and Random Forest. Training and testing areas were identified on this image and which were later used on multidate S-1 SLC image analysis.

\section{Landsat 5TM Data analysis}

Classification of the Landsat 5TM (with bands 4, 3,2) data was conducted with the aim of visualization as shown in
Fig. 5. The following classes were considered: Acacia Forest, Bushed Themada Grassland, Chloris gayana Grassland, Cynodon - Chloris - Themada Grassland, Cynodon niemfluensis Grassland, Cynodon niemfluensis - wooded Acacia grasslands, Euphorbia Candelabra, Lake Nakuru, Olive $\&$ Teclea Forest, Sand \& Mudflats, Sedges \& Marshes and Tachonandus Bushland. These classes were then compared to the classified image previously generated by ( $\mathrm{Ng}$ 'weno et al., 2010) shown in Fig. 4.

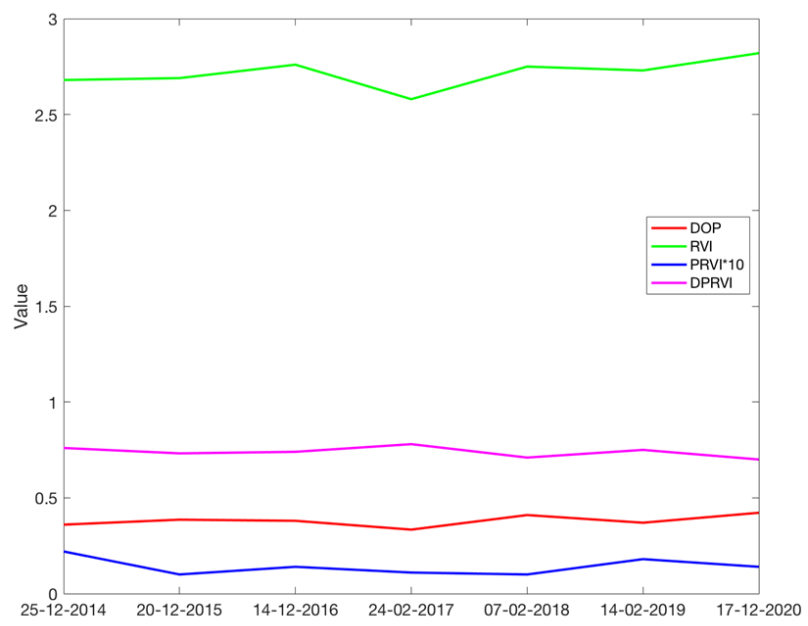

Figure 1: Sentinel 1 SLC spectral response of RVI, DpRVI, PRVI and DOP of the degraded Riparian Reserve 2014-2020 of Lake Nakuru, Kenya.

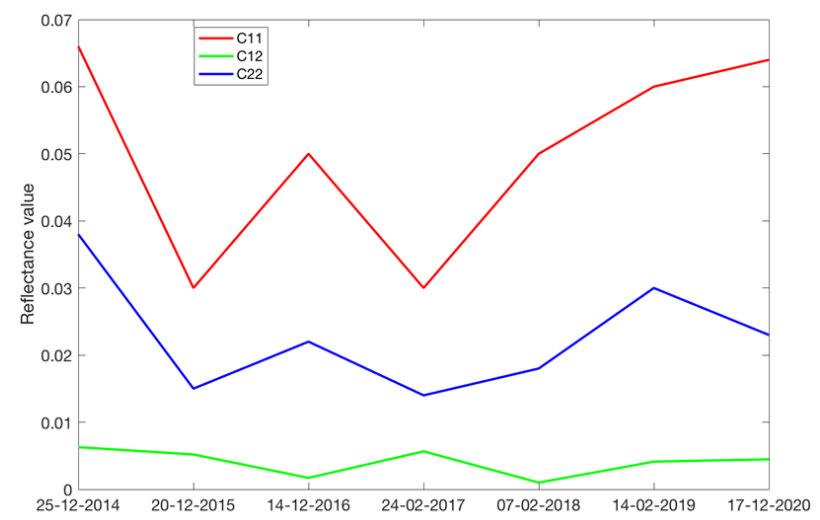

Figure 2: Sentinel 1 SLC covariance matrix generated band averages of $\mathrm{C} 11, \mathrm{C} 12$ and $\mathrm{C} 22$ response on the degraded Riparian Reserve 2014-2020 of Lake Nakuru, Kenya.

\section{RESULTS}

The proposed methodology was designed to fill the following knowledge gaps: i) to determine the spectral changes that have taken place along the riparian reserve by using preprocessed and co-registered multi-date stack time series images built from S-1 Single Look Complex SAR data; ii) to explore the capability of the $\mathrm{C} 2$ covariance matrix derivatives (i.e. C11, C22 and RVI) to characterize Acacia xanthophloea along Lake Nakuru riparian reserve; iii) to explore the spatio-temporal changes that have occurred on the Acacia strands from 2015 to 2020 . We report here the experiments conducted to answers these questions and provide some quantitative and qualitative results. 
3.1 Determine polarimetric spectral response on time series images detected along the riparian reserve via temporal profiles

We generate $2 * 2$ covariance matrix from SLC data to enhance polarimetric characterization of the target. The covariance matrix (C2) data (header and binary files) are used in the generation of C11, C22, C12REAL and C12IMAG bands. We derive radarbased vegetation indices, namely Radar Vegetation Indices (RVI) (Mandal et al., 2020b), Dual Pol Vegetation Index (DpRVI), Polarimetric Radar Vegetation Index (PRVI) (Mandal et al., 2020a) and Degree of Polarization (DOP) for Dual Polarimetric SAR. The bands that had the highest backscatter response, i.e. C11, C22 and RVI were then selected for multi-date image classification across time series of Sentinel 1 Single Look Complex data captured from the period 2014-2020. Fig.1 and Fig. 2 show the multi-temporal average backscatter response of the $\mathrm{C} 2(2 * 2)$ covariance matrix generated bands.

\subsection{The capability of $\mathrm{C} 2$ covariance matrix and spectral deriva-} tives in detecting the changes on the riparian vegetation

In this section the C11, C22 and RVI bands of each imagery captured after the floods between 2014 and 2020 are classified in eCognition Developer 10.1 environment. For further class refinement, the shapefiles consisting of the resulting classes, namely Acacia Forest, Acacia undergrowth, Acacia crown gap and Other were exported to ARCGIS 10.5. Later the tabular CSV files consisting of multi-date Sentinel 1 SLC datasets were transited to WEKA, an open source machine learning environment.

We report below the OBIA classification results conducted on the Acacia Strands along the different parts of Lake Nakuru (i.e. north-western and southern) at three different dates, namely December 2015, February 2017 and February 2019.

North-western, 2015. We consider both a multi-resolution segmentation with parameters set to scale $=5$, shape $=0.6$ and compactness $=0.8$ (Kavzoglu and Tonbul, 2017), and a spectral difference segmentation with scale $=10$ (Yang et al., 2017). Indeed, using multiple segmentation algorithms has been proven to be lead to better results (Tonbul and Kavzaoğlu, 2019). Features included in the classification were the mean and standard deviation of C11, C22 and RVI. As far as geometric features are concerned, we selected shape index, rectangular fit, roundness and ecliptic fit (Kaplan and Avdan, 2019). The default K-NN classifier yielded an overall accuracy (OA) of $87 \%$ and a Cohen's Kappa score of 0.78 . Using Random Forest with baggings and 100 iterations over 737 instances and 12 attributes with a 2/3-1/3 train-test split yielded higher values, with $96.1 \%$ of OA and 0.93 for Kappa. Cross-validation with a 10-fold approach improves further the results, with an OA of $96.7 \%$ and a Kappa of 0.94 . Out of the 737 instances in the classification, 494 were detected as belonging to the Acacia Forest class while 18 fall into the class Other.

Southern, 2015. For this second experiment, we rely on multiresolution segmentation with scale $=10$, shape $=0.6$ and compactness $=0.8$, and give more weight to bands $\mathrm{C} 11$ and RVI as shown on Fig. 1 and Fig. 2. The mean and standard deviation of C11, $\mathrm{C} 22$ and RVI were also included in the classification. Geometric features such as shape index, ecliptic fit, roundness and rectangular fit were completing the set of features. The default K-NN algorithm in eCognition environment yielded an OA of $83 \%$ and a kappa of 0.76. Comparatively, the Random Forest classification with a 10-fold cross validation yielded an OA of $96.3 \%$ and a Kappa of 0.94, this time lower than the 2/3-1/3 train-test split yielding an OA of $96.8 \%$ and a Kappa of 0.95 . Out of the 2491 instances used in the classification, 711 instances were classified as Acacia Forest while 6 instances were confused as class Other. True positive rate (TPR) and false positive rate (FPR) for Acacia Forest were measured as $99.7 \%$ and $0.3 \%$ respectively.

North-western, 2017. Again, we used multi-resolution segmentation with scale $=10$, shape $=0.6$ and compactness $=0.8$ (yielded 1014 objects), as well as spectral difference segmentation at scale 10 , and consider the mean and standard deviation of C11, C22 and RVI, and integrate a set of geometric features (shape index, rectangular fit, roundness, border index and ecliptic fit). We obtain an OA of $97 \%$ and a Kappa of 0.95 with the default KNN classifier, while Random Forest classification carried out in WEKA environment using baggings with 100 iterations and 10fold cross-validation yielded an OA of $94.4 \%$ and a Kappa coefficient of 0.91 . Out of the 1,140 instances used in this classification, 232 instances were classified as Acacia Forest while 22 instances confused as class Other. TPR for the Acacia Forest target class was $91 \%$ while FPR reaches $2.5 \%$. Using the $2 / 3-1 / 3$ train test split lowers the rsults, with an OA of $91.5 \%$ and Kappa equals to 0.86 . Out of the 388 test instances, 64 were classified as Acacia Forest while 11 as class Other.

Southern, 2017. We continue to use C11, C22 and RVI bands, but also apply a Principal Component Analysis (PCA) leading to 3 principal components, i.e. PCA1, PCA2 and PCA3. We then replace $\mathrm{C} 22$ by PCA1 and reorder the bands as C11, RVI, and PCA1, before applying a multi-resolution segmentation with scale $=10$, shape $=0.6$ and compactness $=0.8$, and a spectral difference segmentation at scale 10 to separate class Acacia Forest from Sporobolus Spicatus and Chloris gayana grasslands. KNN classifier yielded an OA of $98.7 \%$ and a Kappa of 0.97 . Let us emphasize the positive role played by the PCA textural features in the classification process, with an improvement of $15.4 \%$ w.r.t. North-western, 2015 (where no such features were used). Comparatively, Random Forest classification with 10-fold crossvalidation yielded an OA of $97.4 \%$ and a Kappa of 0.96 . Out of the 2668 instances used in this approach, 1250 were classified as Acacia Forest and 3 belong to class Acacia undergrowth, 17 in Other. TPR stood at $97.8 \%$ while FPR was $2.2 \%$. Finally, the $2 / 3$ $1 / 3$ train-test split yielded an OA of $98.1 \%$ and a Kappa of 0.97 . 1249 test instances were classified as Acacia Forest while 11 instances were confused as either Acacia Undergrowth or Other. TPR and FPR were measured to $98.8 \%$ and $1.2 \%$, respectively.

North-western, 2019. Similarly to the previous case, we consider C11, C22, RVI, and the Principal Components that can be derived from them. Here, we give more weight to C11, RVI, PCA1 and PCA2 according to their importance as determined by their correlation coefficient (e.g. $R=0.91$ between PCA2 and RVI, $R=0.97$ between $\mathrm{C} 11$ and PCA1, and $R=0.96$ between C22 and PCA2). Some geometric features, namely ecliptic fit, shape index, rectangular fit, roundness, border index and shape index were used. Best results were obtained with superpixel segmentation (SLICO) (Mi and Chen, 2020) and spectral difference segmentation. The default K-NN classifier in eCognition Developer 10.5 yielded perfect results (OA of $100 \%$, Kappa of 1.0). Random Forest bagging with 100 iterations and 10-fold cross-validation yielded an OA of $97.9 \%$ and a Kappa of 0.96 . However TPR of the target class (Acacia Forest) achieved stood at $85.4 \%$ while FPR stood at $1.3 \%$. Out of the 1634 instances, 70 were classified as Acacia Forest while 21 instances were misclassified as either class Other or Acacia undergrowth. The 2/3-1/3 train-test split yielded an OA of $98.3 \%$ and a Kappa of 0.97, leading to TPR and FPR for the Acacia Forest class measured at $79 \%$ and $0.5 \%$, respectively. Out of the 624 test instances, 22 were 
classified as Acacia Forest while 3 instances were misclassified as Acacia undergrowth.

Southern, 2019 The same approach and parameters used in the previous setup were considered here: bands C11, C22, RVI, PCA1, PCA2, PCA3; their mean and standard deviation; superpixel (SLICO) and spectral difference segmentation. K-NN achieves again perfect results. Random Forest with baggings, 100 iterations and 10-fold cross-validation realised an OA of $86.1 \%$ and a Kappa of 0.78 . Out of the 1014 instances, 141 were correctly classified as Acacia Forest, 21 misclassified as Acacia undergrowth, 8 as Other.TPR for the target class (Acacia Forest) was $90 \%$ and FPR was $4 \%$. Random Forst with a 2/3-1/3 traintest split resulted in OA of $86.7 \%$ and Kappa of 0.79 . Out of the 345 instances involved in this classification, 54 instances were assigned the target class Acacia Forest while 2 were misclassified as class Other and 8 as class Acacia undergrowth. TPR and FPR for this target class were then $98 \%$ and $5 \%$.

\subsection{Spatio-Temporal Changes on Acacia Xanthophloea spp Along Lake Nakuru Riparian Reserve}

We have carried out statistical change detection on the different training and test areas. This was made possible by calculating the area of the Acacia X. Spp strands not affected by the floods ranging from 2014 to 2020 as shown on the bar graphs in Figure 3. Site 1 corresponds to the Northern side of Lake Nakuru, divided in a training site (north-western, in blue in the figure) and a test site (north-eastern, in yellow in the figure). Similarly, site 2 corresponds to the Southern side of the lake (with the same color code). Figure 3 reports the distribution of Acacia Forest strands in the different training and test sites.

In site 1 (train), the spatial difference in Acacia X. Spp strands between year 2014 and 2020 was accounting for an area of 6236 pixels, corresponding to an area initially covered by the Healthy Acacia Forest that became degraded and submerged in the flooded lake. For the test area, the difference in area covered by the healthy Acacia X. Spp between year 2014 and 2020 was 22,552 pixels as of 26th July, 2020. Similarly, the Acacia forest transition for the training area of site 2 (southern part of the Lake Nakuru) consists in a reduction of a total area initially covered by Healthy Acacia X. Spp in 2014 of 43,100 pixels to an area of 20,548 pixels by the year 2020 , i.e. a loss in area of 22,522 pixels. As far as the test site is concerned, we observed a similar loss, from 9,092 pixels in February, 2014 to 963 pixels by July, 2020. In other words, the area covered by degraded Acacia X. Spp accounted for 8,129 pixels.

To determine if the Acacia Forest degradation was actually affected by the floods, the area covered by the lake per each given year (2014-2020) was calculated and correlated to the per pixel area covered by the remnants of the Acacia along Lake Nakuru Riparian Reserve. To do so, we used multi-resolution and spectral difference segmentation approaches on a composite image made of $\mathrm{C} 11, \mathrm{C} 22$, and $\mathrm{C} 11 / \mathrm{C} 22$ bands. We obtained the following measures: $52.2 \mathrm{~km}^{2}$ in $2014,54.11 \mathrm{~km}^{2}$ in $2016,57.24 \mathrm{~km}^{2}$ in 2018 , and $59.8 \mathrm{~km}^{2}$ in 2020 . These figures were correlated to the area covered by the Acacia Forest on train site 1 as follows: 16,528 pixels in $2014,13,320$ pixels in $2016,10,650$ pixels in 2018 , and 10,302 pixels in 2020 . We then use the correlation coefficient to measure strength of relationships between variables. We observed a strong negative correlation of $R=-0.94$ between the area of the Lake against the area covered by Healthy Acacia Forest per each given year. The equation to the regression line in relation to this correlation being $y=57870-808.9 x$. This means that, as the level of the lake continued to rise over the years, the more the Acacia Forest continued to degrade, confirming reports by (Mutangah, 1994) that the main cause of stress and eventual death of the Acacia Xanthophloea was due to the rising water table in a closed drainage system (i.e. Lake Nakuru Basin).

\section{DISCUSSION}

The study demonstrates how S1 captured in SLC mode was used to characterize the degrading Acacia X. Spp along the shores of the flooded Lake Nakuru Riparian reserve. The bands that had the highest reflectance were C11, C22 and RVI. They were used to carry out classifications in an object- based image analysis (OBIA) pipeline using multi-date datasets ranging from 2014 to 2020. We hypothesized that SAR polarimetric bands filtered through the $\mathrm{C} 2(2 * 2)$ Covariance Matrix could improve characterization of the flooded riparian vegetation. In this study, classification was carried out on a time series of SLC products, after which they were imported into eCognition Developer software environment for object detection and classification. Rulesets where different segmentation types and scales (Nico et al., 2000, Yang et al., 2017, Kavzoglu and Tonbul, 2017) were appended on 28 training and testing sites.

The variables that contributed well to the classifications were determined by measuring correlation coefficients. It was worth noting that there was a strong positive correlation between the ecliptic fit and rectangular fit $(R=0.91)$, and between border index and shape index $(R=0.95)$. We follow previous studies that show some improvement of classification accuracy using shape and geometric variables (Kaplan and Avdan, 2019). Other variables that contributed to the high-classification accuracies were the standard deviation of polarimetric bands C11, C22 and RVI, and their principal components. Strong positive correlations were noted between the stdev of C11/PCA1 $(R=0.97), \mathrm{C} 22 / \mathrm{PCA} 2$ $(R=0.96)$ and PCA2/RVI $(R=0.98)$. The inclusion of the texture features (here PCA) onto the classification process increased the K-NN based classification from $15.4 \%$. These results corroborate with previous studies where supervised classification and textural variables have been used to improve classification accuracy (Granata et al., 2020, Osio et al., 2020, Pham et al., 2017).

The difference in the performance of classification algorithm have been demonstrated using different methods. (Gašparović and Dobrinić, 2020) used McNemar's $\chi^{2}$ test to compare the performance of classifier algorithms, including XGB, SVM and Random Forest (RF). In this study we also compare the performance of the three classifiers, namely Naive Bayes, Decision Tree (DT) and Random Forest (RF) in a total of 84 classifications carried out across the multi-date images. Let us note that the qualitative and quantitative analysis provided in the previous section was focusing solely on KNN and RF for the sake of concision. Results achieved through inter-group comparisons using one way ANOVA depicted statistically significant difference between the three classifiers in all the training sites that were used in this study using both cross-validation and 2/3-1/3 train-test split. Random Forest was confirmed as the best performing classifier.

\section{CONCLUSION}

This study demonstrates how Sentinel-1 SLC polarimetric bands $\sigma(\mathrm{VV})$ and $\sigma(\mathrm{VH})$ filtered through covariance matrix could improve wetland classification. We consider an OBIA-based approach coupled with PCA-derived texture features, as well as geometric and shape features.

We have conducted numerous experiments on several datasets and several dates, assessing the effect of all components in the 

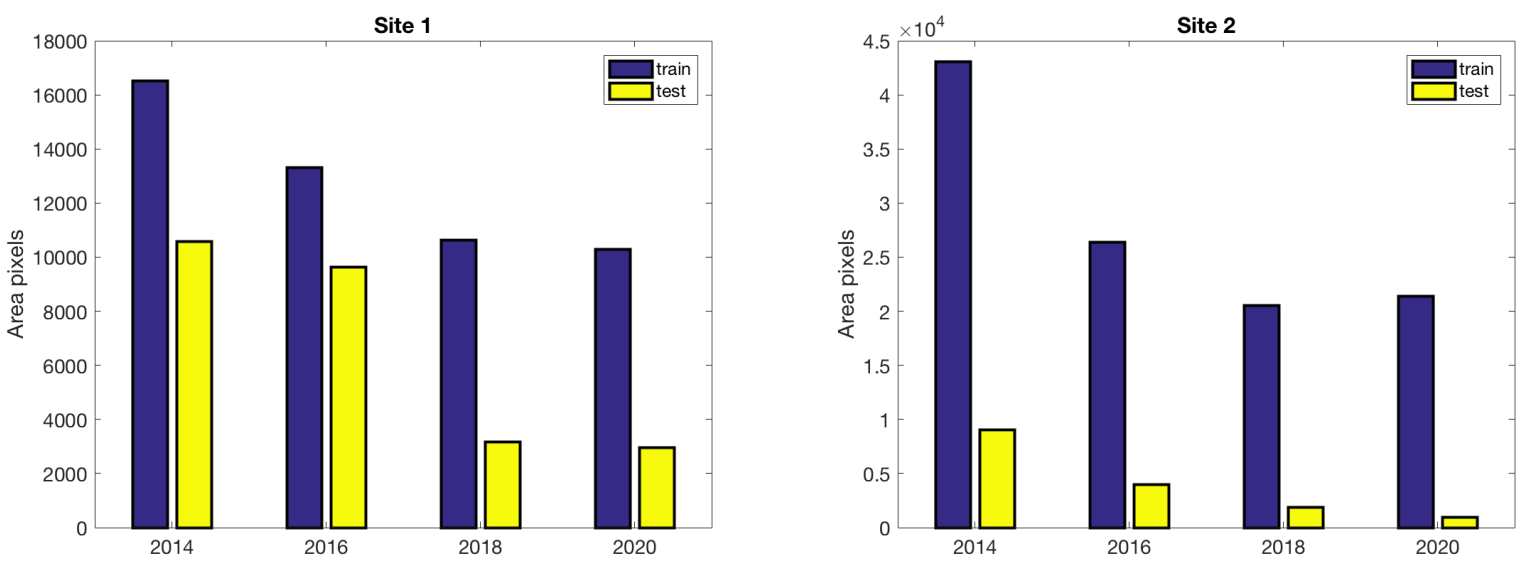

Figure 3: Spatio-Temporal Change Detection on Acacia X. Spp on Training and Test sites 1 (left) and 2 (right).

pipeline: input bands for the segmentation, segmentation algorithm and parameters, features to describe the objects, and classifier settings. We have observed that the optimal selection was specific to each image (area, date) under study. The average results achieved by ALOS PALSAR with Random Forest (OA of 93.8\%, Kappa of 0.92) makes it a suitable choice as a reference image in this study. Out of 84 classifications carried out, Random Forest (RF) (Zamani Joharestani et al., 2019) achieved the highest average score (OA of $90.0 \%$, Kappa of 0.88). However in some instances, Acacia Forest was either confused as class Acacia Undergrowth or Other, hence confirming reports by (Moskal et al., 2011) on confusion between classes in wetland-based land cover classification.

Between 2014 and 2020 the area covered by the degraded Acacia $\mathrm{X}$. Spp trees on the north western side of the lake was 6,236 pixels, on the southern part where the largest strands of acacia forest were existing 22,522 pixels, and on the south western side 8,129 pixels were degraded. There was a strong negative correlation ( $R=-0.94$ ) between the area covered by the lake against the area covered by the Acacia Forest remnants on the riparian reserve, confirming reports by (Mutangah, 1994) that the flooding lake could have been the cause of the death of the Acacia Trees within the closed ecosystem. Future studies should look into the possibility of using Quad polarized Sentinel 1 SLC products to characterize flooded riparian vegetation.

\section{ACKNOWLEDGEMENTS}

The authors acknowledge: Kenya National Research Fund (K$\mathrm{NRF}$ ) and Campus France through Pamoja PHC programme; European Space Agency for the provision of Sentinel-1 Products; Trimble Inc. Germany for the provision of eCognition Developer Software Licence.

\section{REFERENCES}

Amani, M. and Mobasheri, M. R., 2015. A parametric method for estimation of leaf area index using landsat etm+ data. GIScience \& Remote Sensing 52(4), pp. 478-497.

Anusha, N. and Bharathi, B., 2020. Flood detection and flood mapping using multi-temporal synthetic aperture radar and optical data. The Egyptian Journal of Remote Sensing and Space Science 23(2), pp. 207-219.

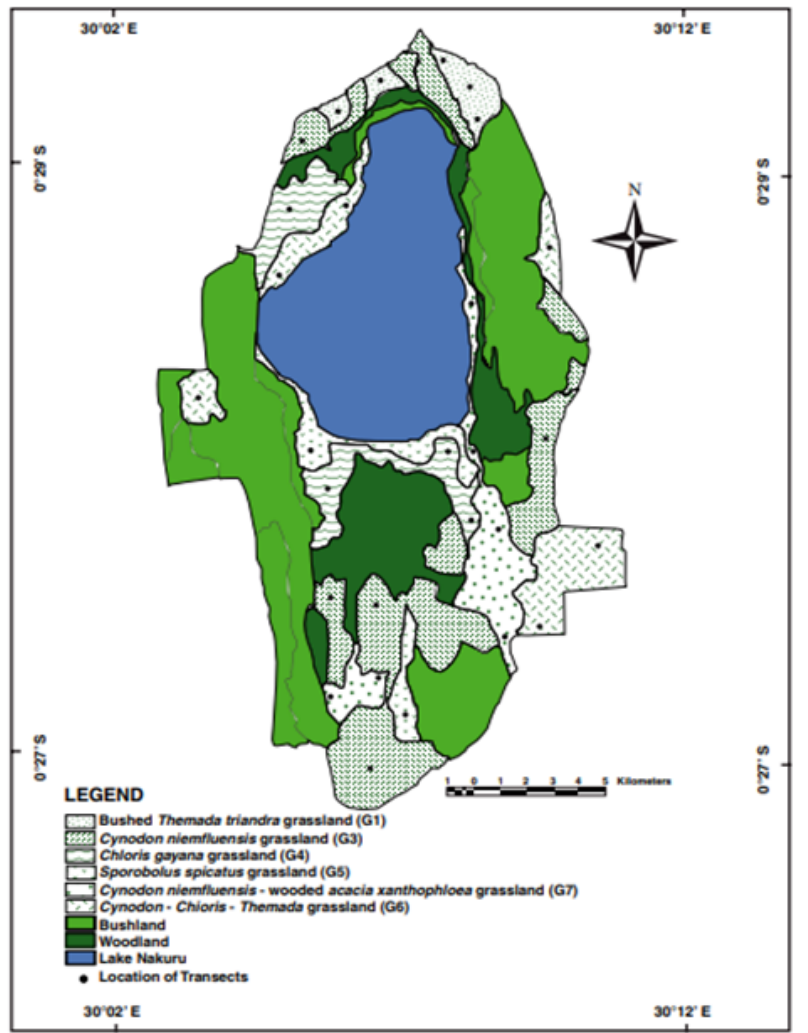

Figure 4: Distribution of vegetation classes in Lake Nakuru National Park (illustration from (Ng'weno et al., 2010)).

Franklin, S. E. and Ahmed, O. S., 2017. Object-based wetland characterization using radarsat-2 quad-polarimetric sar data, landsat- 8 oli imagery, and airborne lidar-derived geomorphometric variables. Photogrammetric Engineering \& Remote Sensing 83(1), pp. 27-36.

Gašparović, M. and Dobrinić, D., 2020. Comparative assessment of machine learning methods for urban vegetation mapping using multitemporal sentinel-1 imagery. Remote Sensing 12(12), pp. 1952.

Granata, F., Gargano, R. and de Marinis, G., 2020. Artificial intelligence based approaches to evaluate actual evapotranspiration in wetlands. Science of The Total Environment 703, pp. 135653.

Kaplan, G. and Avdan, U., 2019. Evaluating the utilization of 


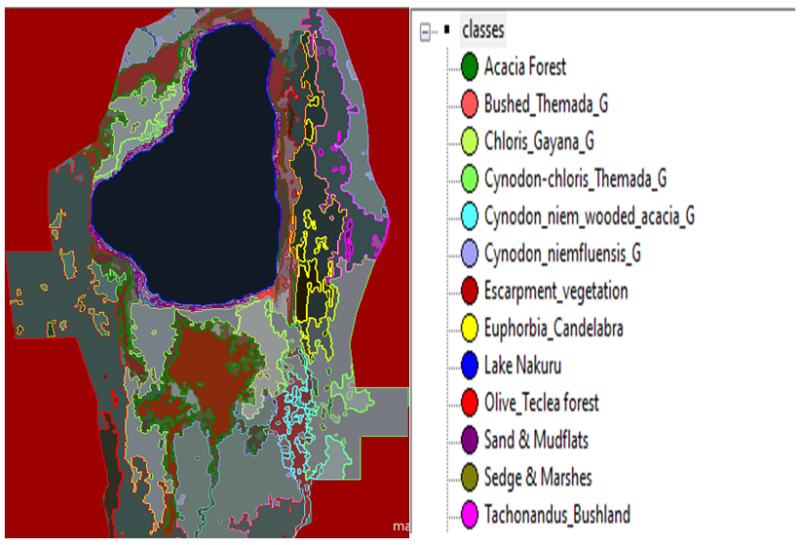

Figure 5: Landcover classification in the study area, before the onset of flooding.
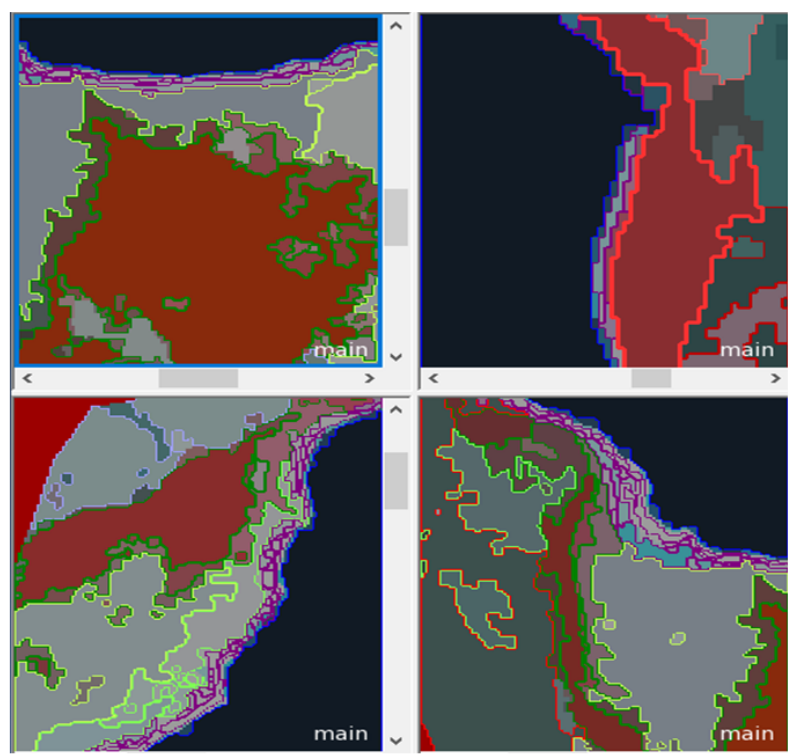

Figure 6: The state of Acacia forest patches clockwise from upper left corner: Southern part, North-Eastern part, Southern-Western part, \& North-Western part of the study area where Acacia Forest patches (in red) existed before the onset of flooding.

the red edge and radar bands from sentinel sensors for wetland classification. Catena 178, pp. 109-119.

Kavzoglu, T. and Tonbul, H., 2017. A comparative study of segmentation quality for multi-resolution segmentation and watershed transform. In: 2017 8th International Conference on Recent Advances in Space Technologies (RAST), IEEE, pp. 113-117.

Lee, J.-S. and Pottier, E., 2017. Polarimetric radar imaging: from basics to applications. CRC press.

Mandal, D., Kumar, V., Ratha, D., Dey, S., Bhattacharya, A., Lopez-Sanchez, J. M., McNairn, H. and Rao, Y. S., 2020a. Dual polarimetric radar vegetation index for crop growth monitoring using sentinel-1 sar data. Remote Sensing of Environment 247, pp. 111954.

Mandal, D., Ratha, D., Bhattacharya, A., Kumar, V., McNairn, H., Rao, Y. S. and Frery, A. C., 2020b. A radar vegetation index for crop monitoring using compact polarimetric sar data. IEEE Transactions on Geoscience and Remote Sensing 58(9), pp. 6321-6335.
Mi, L. and Chen, Z., 2020. Superpixel-enhanced deep neural forest for remote sensing image semantic segmentation. ISPRS Journal of Photogrammetry and Remote Sensing 159, pp. 140152.

Moskal, L. M., Styers, D. M. and Halabisky, M., 2011. Monitoring urban tree cover using object-based image analysis and public domain remotely sensed data. Remote Sensing 3(10), pp. 22432262.

Mutangah, J. G., 1994. The vegetation of lake nakuru national park, kenya: a synopsis of the vegetation types with annotated species list. Journal of East African Natural History 83(1), pp. 71-96.

Ng'weno, C. C., Mwasi, S. M. and Kairu, J. K., 2010. Distribution, density and impact of invasive plants in lake nakuru national park, kenya. African Journal of Ecology 48(4), pp. 905-913.

Nico, G., Pappalepore, M., Pasquariello, G., Refice, A. and Samarelli, S., 2000. Comparison of sar amplitude vs. coherence flood detection methods-a gis application. International Journal of Remote Sensing 21(8), pp. 1619-1631.

Onywere, S., Shisanya, C., Obando, J., Ndubi, A., Masiga, D., Irura, Z., Mariita, N. and Maragia, H., 2013. Geospatial extent of 2011-2013 flooding from the eastern african rift valley lakes in kenya and its implication on the ecosystems. In: Papers, Kenya Soda Lakes workshop. Kenya Wildlife Service Training Institute, Naivasha.

Osio, A., Pham, M. and Lefèvre, S., 2020. Spatial processing of sentinel imagery for monitoring of acacia forest degradation in lake nakuru riparian reserve. ISPRS Annals of the Photogrammetry, Remote Sensing and Spatial Information Sciences 3, pp. 525532.

Pellew, R., 1983. The giraffe and its food resource in the serengeti. i. composition, biomass and production of available browse. African Journal of Ecology 21(4), pp. 241-267.

Pham, M.-T., Lefevre, S. and Aptoula, E., 2017. Local featurebased attribute profiles for optical remote sensing image classification. IEEE Transactions on Geoscience and Remote Sensing 56(2), pp. 1199-1212.

Schumann, G., Di Baldassarre, G., Alsdorf, D. and Bates, P., 2010. Near real-time flood wave approximation on large rivers from space: Application to the river po, italy. Water Resources Research.

Tonbul, H. and Kavzaoğlu, T., 2019. Application of taguchi optimization and anova statistics in optimal parameter setting of multi-resolution segmentation. In: 2019 9th International Conference on Recent Advances in Space Technologies (RAST), IEEE, pp. 387-391.

Yang, J., He, Y. and Caspersen, J., 2017. Region merging using local spectral angle thresholds: A more accurate method for hybrid segmentation of remote sensing images. Remote sensing of environment 190, pp. 137-148.

Zamani Joharestani, M., Cao, C., Ni, X., Bashir, B. and Talebiesfandarani, S., 2019. Pm2. 5 prediction based on random forest, xgboost, and deep learning using multisource remote sensing data. Atmosphere 10(7), pp. 373. 